EXTENDED REPORT

\title{
Correlation between retinal nerve fibre layer thickness and optic nerve head size: an optical coherence tomography study
}

\author{
G Savini, M Zanini, V Carelli, A A Sadun, F N Ross-Cisneros, P Barboni
}

Br J Ophthalmol 2005;89:489-492. doi: 10.1136/bjo.2004.052498

See end of article for authors' affiliations

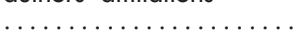

Correspondence to: Giacomo Savini, Centro Salus, via Saffi $4 \mathrm{H}, 40131$ Bologna, Italy; giacomo.savini@ fastwebnet.it

Accepted for publication 1 September 2004

\begin{abstract}
Aim: To investigate the correlation between retinal nerve fibre layer (RNFL) thickness and optic nerve head $(\mathrm{ONH})$ size in normal white subjects by means of optical coherence tomography (OCT).

Methods: 54 eyes of 54 healthy subjects aged between 15 and 54 underwent peripapillary RNFL thickness measurement by a series of three circular scans with a $3.4 \mathrm{~mm}$ diameter (Stratus OCT, RNFL Thickness 3.4 acquisition protocol). $\mathrm{ONH}$ analysis was performed by means of six radial scans centred on the optic disc (Stratus OCT, Fast Optic Disc acquisition protocol). The mean RNFL values were correlated with the data obtained by $\mathrm{ONH}$ analysis.

Results: The superior, nasal, and inferior quadrant RNFL thickness showed a significant correlation with the optic disc area $(R=0.3822, p=0.0043),(R=0.3024, p=0.026),(R=0.4048, p=0.0024)$ and the horizontal disc diameter $(R=0.2971, p=0.0291),(R=0.2752, p=0.044),(R=0.3970, p=0.003)$. The superior and inferior quadrant RNFL thickness was also positively correlated with the vertical disc diameter $(R=0.3774, p=0.0049),(R=0.2793, p=0.0408)$. A significant correlation was observed between the $360^{\circ}$ average RNFL thickness and the optic disc area and the vertical and horizontal disc diameters of the ONH ( $R=0.4985, p=0.0001),(R=0.4454, p=0.0007),(R=0.4301, p=0.0012)$.

Conclusions: RNFL thickness measurements obtained by Stratus OCT increased significantly with an increase in optic disc size. It is not clear if eyes with large ONHs show a thicker RNFL as a result of an increased amount of nerve fibres or to the shorter distance between the circular scan and the optic disc edge.
\end{abstract}

A lthough clinical examination of the optic nerve head $(\mathrm{ONH})$ is still considered to be the most sensitive method for identifying glaucomatous damage, new ocular imaging technologies such as optical coherence tomography (OCT) are gaining popularity in the diagnosis of glaucoma. They enable clinicians to perform accurate, objective, and reproducible measurements of the retinal nerve fibre layer (RNFL) and ONH topography. ${ }^{1}$

OCT is a high resolution reproducible imaging technology that has been widely used to evaluate the RNFL in patients with or without glaucoma. ${ }^{2}$ Images are obtained by delivery of low coherence near infrared light $(850 \mathrm{~nm})$ from a super luminescent diode and subsequent backscattering from the retina. Recent software enhancement also allows $\mathrm{ONH}$ analysis. ${ }^{13}$

To assess RNFL thickness, a circular scan concentric to the ONH is performed. In 1996 Schuman and co-workers found a circle diameter of $3.4 \mathrm{~mm}$ to be the most accurate as regards reproducibility and all studies since then have used circular scans with this diameter, independently of ONH size. ${ }^{4}$ However, it is generally recognised that the optic disc size shows a high interindividual variability in normal eyes and its area may range between 0.8 and $6.0 \mathrm{~mm}^{2} .5$ Similarly, a high variability has been found in other $\mathrm{ONH}$ parameters, such as the optic disc vertical (range $0.96-2.91 \mathrm{~mm}$ ) and horizontal (range 0.91-2.61 mm) diameters and neuroretinal rim size (range $0.8-4.66 \mathrm{~mm}^{2}$ ). ${ }^{6}$ As a consequence, using the same, fixed diameter circular scan in all eyes may result in RNFL thickness measurements performed at different distances from the ONH margin. Since histological studies have already demonstrated that RNFL thickness decreases with increasing distance from the disc margin, ${ }^{7}$ we were prompted to investigate how RNFL thickness, as measured by OCT, may be influenced by the optic disc size and thus by the distance between the circular scan and the $\mathrm{ONH}$ margin.

\section{PATIENTS AND METHODS \\ Patients}

A total of 54 eyes of 54 normal white subjects aged between 15 and 54 were enrolled in this study (23 males and 31 females, mean age 34 (SD 10.3)). The study population consisted of volunteers from the staff of Centro Salus and patients with minor refractive disorders. Before being included in the study (that had been approved by the institutional review board of Centro Salus, Bologna, Italy) each subject was informed of its purpose and gave written consent to participate. Subjects ranged in age from 15 to 54; patients older than 55 were intentionally excluded to minimise the influence of age and lens opacities on RNFL thickness measurement. ${ }^{8}$ Inclusion criteria were the following: best corrected visual acuity above 20/25, refractive error between -5 and +5 dioptres of sphere or between -2 and +2 dioptres of cylinder, normal intraocular pressure $<21 \mathrm{~mm} \mathrm{Hg}$, normal appearance of the optic disc, no significant ocular disease found by routine ophthalmological examination, no history of glaucoma in the family, and/or systemic diseases with possible ocular involvement, such as diabetes mellitus. In addition, patients had reliable measurements of visual field (fixation loss $<20 \%$, false negative and false positive $<25 \%$, mean deviation and corrected pattern standard deviation within 95\% normal limits, and a glaucoma hemifield test result within normal limit) performed using the central 24-2 or 30-2 program of the

Abbreviations: $\mathrm{OCT}$, optical coherence tomography; $\mathrm{ONH}$, optic nerve head; RNFL, retinal nerve fibre layer 
Humphrey visual field analyser (Allergan-Humphrey, San Leandro, CA, USA).

\section{OCT measurements}

OCT 3000 (Stratus OCT, software version 3.0; Carl Zeiss Meditec, Inc, Dublin, CA, USA) was used to measure both the thickness of the peripapillary RNFL and ONH size. The examination was performed under mydriasis by two experienced operators (GS and PB).

Stratus OCT employs low coherence interferometry to generate cross sectional images of the retina, optic disc and RNFL with $\leqslant 10 \mu \mathrm{m}$ axial resolution and transverse resolution of $20 \mu \mathrm{m}$. The instrument contains an interferometer that resolves posterior pole structures by measuring the echo delay time of light that is reflected and backscattered from different layers in the retina and optic disc. The RNFL thickness algorithm searches for the RNFL in a two pass process: it looks first for the highest rates of changes in reflectivity at the vitreoretinal interface and then for reflectivity above a threshold value in its adjacent highly reflective layer. The threshold is individually determined for each scan as a multiple of the local maximum reflectance to adjust for variations in optical alignment or drying of the corneal surface or changes in pupil size. The nerve fibre layer thickness is defined as a multiple of the number of pixels between the anterior and posterior edges of the RNFL.

To assess RNFL thickness, measurements were made along a circle concentric with the optic disc at a radius of $1.73 \mathrm{~mm}$, using a scanning mode that samples 512 data points (RNFL Thickness 3.4 acquisition protocol). Three measurements were performed for each eye and the mean values were recorded. For each eye, we studied the mean RNFL thickness $\left(360^{\circ}\right.$ measure), temporal quadrant thickness $\left(316^{\circ}-45^{\circ}\right.$ unit circle), superior quadrant thickness $\left(46^{\circ}-135^{\circ}\right)$, nasal quadrant thickness $\left(136^{\circ}-225^{\circ}\right)$, and inferior quadrant thickness $\left(226^{\circ}-315^{\circ}\right)$, all automatically calculated by OCT using the existing software.

Optic nerve head evaluation consisted of six radial scans centred on the ONH, spaced 30 degrees apart (Fast Optic Disc acquisition protocol). Each radial scan included 128 points. The machine automatically defined the edge of the optic disc as the end of the retinal pigment epithelium/choriocapillaris and used smoothing with fit to circle to fill the gaps between scans. The resultant image could be manually corrected when the machine did not identify the edge correctly. A straight line connected the edges of the retinal pigment epithelium/ choriocapillaris and a parallel line was constructed $150 \mu \mathrm{m}$ anteriorly. Structures below this line were defined as the disc cup and above this line as the neuroretinal rim. Among the measurements given by the OCT optic nerve head analysis, the following were examined: disc area, cup area, rim area, cup/disc vertical and horizontal ratios, vertical and horizontal disc diameters.

The OCT data were exported to a personal computer, and left eye data were converted into right eye format. Only good quality OCT data as judged by the appearance of the RNFL and the optic disc pictures were used for further analysis. Images with artefacts, missing parts, or showing seemingly distorted anatomy were excluded. As regards RNFL measurements, since the position of the circular scan with respect to the optic disc is crucial, we included only images where the $\mathrm{ONH}$ was well centred by the scan. In the case of ONH analysis, if necessary, the manual option was used to correct a displaced $\mathrm{ONH}$ margin.

\section{Statistics}

All statistical analyses were performed using GraphPad InStat version 3a for Macintosh (GraphPad Software, San Diego, CA, USA, www.graphpad.com). For statistical evaluations only one eye, randomly chosen, was considered for each patient. Possible relations between the RNFL thickness and ONH parameters were analysed using the Pearson correlation for parametric data and Spearman correlation for nonparametric data. The correlation between the $360^{\circ}$ average RNFL thickness and the $\mathrm{ONH}$ area was selected as the primary end point, to maintain the overall type 1 error rate without $p$ value adjustments. ${ }^{10}$ In addition, multiple regression was performed to exclude age as a confounding factor between each RNFL measurement and all ONH parameters. A p value lower than 0.05 was considered statistically significant.

\section{RESULTS}

Good quality RNFL and ONH images could be achieved in all 54 eyes entering the study; manual correction of ONH edges was necessary in 38 eyes $(61.1 \%)$. All eyes could undergo statistical analysis.

The mean values, standard deviation, range, and 95\% confidence interval of RNFL thickness are reported in table 1 together with the measures of each $\mathrm{ONH}$ parameter considered.

The results of the correlation between RNFL thickness and each optic disc size parameter are reported in table 2. As far as the primary end point is concerned, a positive correlation was found between the $360^{\circ}$ average RNFL thickness and the ONH area $(\mathrm{R}=0.4985 \mathrm{p}=0.0001)$ (fig 1). Positive correlations were also found between:

Table 1 Mean values, standard deviation (SD), range, and $95 \%$ confidence interval (Cl) for each of the RNFL and ONH parameters considered in this study

\begin{tabular}{|c|c|c|c|c|}
\hline & Mean & SD & Range & $95 \% \mathrm{Cl}$ \\
\hline \multicolumn{5}{|l|}{ RNFL } \\
\hline $360^{\circ}$ average $(\mu \mathrm{m})$ & 101.52 & 11.09 & $78.43-130.75$ & 98.49 to 104.56 \\
\hline Temporal quadrant $(\mu \mathrm{m})$ & 69.94 & 13.17 & $42-109$ & 66.34 to 73.54 \\
\hline Superior quadrant $(\mu \mathrm{m})$ & 124.29 & 16.59 & $91-163$ & 119.76 to 128.83 \\
\hline Nasal quadrant $(\mu \mathrm{m})$ & 81.24 & 17.95 & $46-133$ & 76.33 to 86.14 \\
\hline Inferior quadrant $(\mu \mathrm{m})$ & 129.83 & 19.26 & $66-184$ & 124.57 to 135.09 \\
\hline \multicolumn{5}{|l|}{ ONH } \\
\hline Optic disc area $\left(\mathrm{mm}^{2}\right)$ & 2.09 & 0.32 & $1.55-2.94$ & 2 to 2.18 \\
\hline Cup area $\left(\mathrm{mm}^{2}\right)$ & 0.41 & 0.27 & $0-1.24$ & 0.33 to 0.49 \\
\hline Rim area $\left(\mathrm{mm}^{2}\right)$ & 1.67 & 0.33 & $0.85-2.53$ & 1.58 to 1.77 \\
\hline Cup/disc vertical ratio & 0.42 & 0.18 & $0-0.71$ & 0.37 to 0.47 \\
\hline Cup/disc horizontal ratio & 0.38 & 0.15 & $0-0.62$ & 0.34 to 0.42 \\
\hline Vertical diameter $(\mathrm{mm})$ & 1.68 & 0.14 & $1.44-2.03$ & 1.64 to 1.72 \\
\hline Horizontal diameter (mm) & 1.55 & 0.14 & $1.32-1.96$ & 1.51 to 1.59 \\
\hline
\end{tabular}


Table 2 Correlation between RNFL thickness and ONH parameters

\begin{tabular}{|c|c|c|c|c|c|c|c|}
\hline RNFL thickness & Optic disc area & Cup area & Rim area & $\begin{array}{l}\text { Vertical } \\
\text { C:D ratio }\end{array}$ & $\begin{array}{l}\text { Horizontal } \\
\text { C:D ratio }\end{array}$ & $\begin{array}{l}\text { Vertical } \\
\text { diameter }\end{array}$ & $\begin{array}{l}\text { Horizontal } \\
\text { diameter }\end{array}$ \\
\hline $360^{\circ}$ average & $\begin{array}{l}R=0.4985 \\
p=0.0001\end{array}$ & $\begin{array}{l}R=0.0951 \\
p=N S\end{array}$ & $\begin{array}{l}R=0.4024 \\
p=0.0026\end{array}$ & $\begin{array}{l}R=0.0879 \\
p=N S\end{array}$ & $\begin{array}{l}R=0.0610 \\
p=N S\end{array}$ & $\begin{array}{l}R=0.4454 \\
p=0.0007\end{array}$ & $\begin{array}{l}R=0.4301 \\
p=0.0012\end{array}$ \\
\hline Temporal quadrant & $\begin{array}{l}R=0.2026 \\
p=N S\end{array}$ & $\begin{array}{l}R=-0.1263 \\
p=N S\end{array}$ & $\begin{array}{l}R=0.2952 \\
p=0.0302\end{array}$ & $\begin{array}{l}R=-0.1537 \\
p=N S\end{array}$ & $\begin{array}{l}R=-0.2067 \\
p=N S\end{array}$ & $\begin{array}{l}R=0.1838 \\
p=N S\end{array}$ & $\begin{array}{l}R=0.1599 \\
p=N S\end{array}$ \\
\hline Superior quadrant & $\begin{array}{l}R=0.3822 \\
p=0.0043\end{array}$ & $\begin{array}{l}R=-0.003 \\
p=N S\end{array}$ & $\begin{array}{l}R=0.3474 \\
p=0.0101\end{array}$ & $\begin{array}{l}R=0.0677 \\
D=N S\end{array}$ & $\begin{array}{l}R=0.0395 \\
p=N S\end{array}$ & $\begin{array}{l}R=0.3774 \\
p=0.0049\end{array}$ & $\begin{array}{l}R=0.2971 \\
p=0.0291\end{array}$ \\
\hline Nasal quadrant & $R=0.3024$ & $R=0.1552$ & $\begin{array}{l}R=0.1274 \\
n=N S\end{array}$ & $\begin{array}{l}\mathrm{R}=0.1485 \\
\mathrm{n}=\mathrm{NS}\end{array}$ & $R=0.1505$ & $\begin{array}{l}\mathrm{R}=0.1490 \\
\mathrm{D}=\mathrm{NS}\end{array}$ & $\mathrm{R}=0.2752$ \\
\hline Inferior quadrant & $\begin{array}{l}R=0.4048 \\
p=0.0024\end{array}$ & $\begin{array}{l}R=0.1160 \\
p=N S\end{array}$ & $\begin{array}{l}R=0.3199 \\
p=0.0184\end{array}$ & $\begin{array}{l}R=0.0424 \\
p=N S\end{array}$ & $\begin{array}{l}R=0.0301 \\
p=N S\end{array}$ & $\begin{array}{l}R=0.2793 \\
p=0.0408\end{array}$ & $\begin{array}{l}\mathrm{R}=0.3970 \\
\mathrm{p}=0.0030\end{array}$ \\
\hline
\end{tabular}

(1) the optic disc area and the RNFL thickness of the superior, inferior, and nasal quadrants

(2) the vertical diameter of the ONH and the RNFL thickness of the superior and inferior quadrants

(3) the horizontal diameter of the $\mathrm{ONH}$ and the RNFL thickness of the superior, inferior, and nasal quadrants

(4) the rim area and the RNFL thickness of the superior, inferior, and temporal quadrants.

Each of the above mentioned $\mathrm{ONH}$ parameters was also positively correlated with the $360^{\circ}$ average RNFL thickness. No correlations were detected in any quadrant between the RNFL thickness and cup area, or between the RNFL thickness and either the vertical or horizontal cup/disc ratios.

According to multiple regression, age did not make a significant contribution $(\mathrm{p}=0.64,0.3,0.23,0.54$, and 0.5 respectively for the $360^{\circ}$ average measurement and the temporal, superior, nasal, and inferior quadrants).

\section{DISCUSSION}

Our study shows that RNFL thickness measured by Stratus OCT is positively correlated with ONH size, as determined by measurements of its area and diameter. Such a correlation could be clearly observed in the superior and inferior quadrants, in the $360^{\circ}$ average measurement and, to a lesser extent, in the nasal quadrant, although in some cases the $\mathrm{R}$ values were just mild to moderate. In the temporal quadrant we detected a similar trend, but statistical significance was not achieved. Two explanations-not mutually exclusivecan be proposed for this correlation.

Firstly, our results are simply confirmatory of previous histological studies showing that the optic nerve fibre count increases with enlarging $\mathrm{ONH}$ size. ${ }^{11}{ }^{12}$ Although these studies analysed the retrobulbar portion of the optic nerve, it may be extrapolated that a similar correlation also exists in

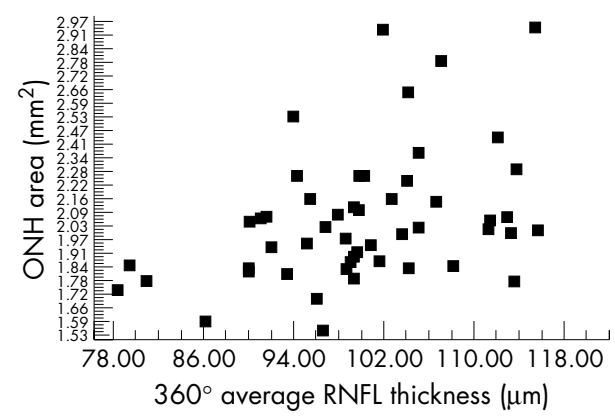

Figure 1 Scatter plot of the primary end point, showing the correlation between $360^{\circ}$ average retinal nerve fibre layer (RNFL) thickness and optic nerve head (ONH) area. the peripapillary RNFL. Additional evidence is provided by the positive correlation found in this study between RNFL thickness (in the superior, inferior, and temporal quadrants and in the $360^{\circ}$ average measurement) and the neuroretinal rim area. The latter correlation is not surprising, since the rim is considered the intrapapillary equivalent of the retinal nerve fibres. From a clinical point of view, it is important to observe that if larger discs really do contain more retinal ganglion cell axons they may benefit from a higher anatomic reserve capacity in progressive optic neuropathies.

Secondly, we may consider our findings in a different light and interpret them as an artefact of the OCT methodology, owing to the fact that the circular scan has a fixed diameter of $3.4 \mathrm{~mm}$, as suggested by previous studies. ${ }^{4}$ It is likely that the positive correlation between the $\mathrm{ONH}$ parameters and RNFL thickness depends on the distance between the OCT

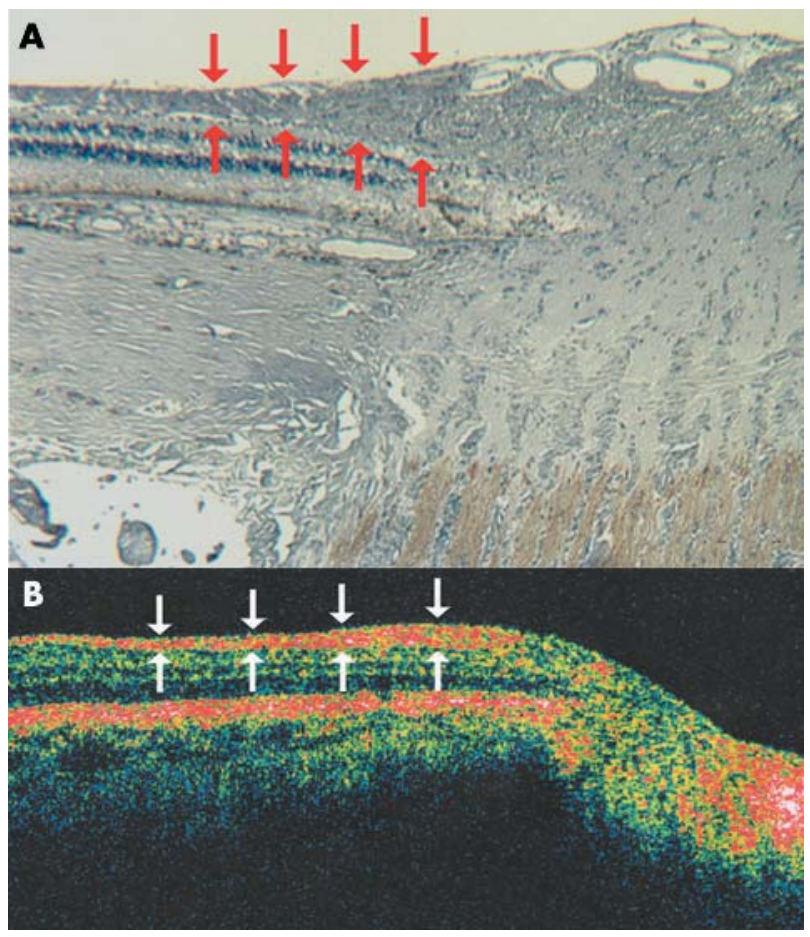

Figure 2 (A) Histological section of the optic nerve head and peripapillary retina, including the retinal nerve fibre layer (RNFL). Red arrows indicate the progressive thinning of the RNFL at increasing distances from the disc margin. (B) Similar section obtained by optical coherence tomography. Again, RNFL thickness decreases (white arrows) as the distance from the optic nerve head increases. Both $A$ and $B$ are horizontal sections from normal human eyes; they were taken from two different subjects. 
circular scan and the ONH margin. RNFL thickness, in fact, has been shown to decrease at increasing distances from the $\mathrm{ONH}$ (fig 2). ${ }^{6}$ If a fixed diameter circular scan is employed, the distance between the scan and the $\mathrm{ONH}$ margin will obviously be reduced in the presence of a large ONH. Such an artefact may lead to an overestimation of RNFL thickness in patients with large ONHs, since the measurements would be made closer to the optic disc edge. Should future studies confirm this hypothesis, it might become necessary to individually adjust the analysis of RNFL thickness according to ONH size; in other words, the diameter of the circular scan should be customised according to optic disc area or diameter. As an alternative, any patient undergoing RNFL analysis by OCT for clinical purposes should be compared to a control group made up of individuals matched for ONH size. Finally, the validity of current OCT databases may be subject to challenge and the data from many, if not all, studies performed using OCT should in any case be carefully reevaluated.

Potential limitations of this study include the fact that multiple statistical tests were performed to investigate the correlation between RNFL and ONH parameters, with no adjustments to $\mathrm{p}$ value (however, to maintain the overall risk of type 1 error-findings of false significance-we selected a primary end point, which resulted highly significant). In addition, although we studied only eyes with a refractive error between -5 and +5 dioptres of sphere or between -2 and +2 dioptres of cylinder, possible artefacts caused by ametropia cannot be fully excluded.

In conclusion, we showed that RNFL thickness as measured by Stratus OCT is positively correlated with ONH size. Such a correlation may be the result of either an increased number of nerve fibres in eyes with larger discs or a smaller distance between the circular scan and the true ONH margin. Further studies (evaluating, for example, the persistence of this correlation after the diameter of the scan has been customised according to ONH size) are needed to verify if one or both of these hypotheses are true.

Authors' affiliations

G Savini, M Zanini, P Barboni, Centro Salus, Bologna, Italy

V Carelli, Dipartimento di Scienze Neurologiche, Università di Bologna, Bologna, Italy

A A Sadun, F N Ross-Cisneros, Doheny Eye Institute and Department of Ophthalmology, Keck School of Medicine of the University of the Southern California, Los Angeles, CA, USA

Proprietary interest: none.

\section{REFERENCES}

1 Paunescu LA, Schuman JS, Price LL, et al. Reproducibility of nerve fiber thickness, macular thickness, and optic nerve head measurement using Stratus OCT. Invest Ophthalmol Vis Sci 2004;45:1716-24.

2 Jaffe GJ, Caprioli J. Optical coherence tomography to detect and manage retinal disease and glaucoma. Am J Ophthalmol 2004;137:156-69.

3 Schuman JS, Wollstein G, Farra T, et al. Comparison of optic disc nerve head measurements obtained by optical coherence tomography and confocal scanning laser ophthalmoscopy. Am J Ophthalmol 2003;135:504-12.

4 Schuman JS, Pedut-Kloizman T, Hertzmark E, et al. Reproducibility of nerve fiber layer thickness measurements using optical coherence tomography. Ophthalmology 1996;103:1889-98.

5 Jonas JB, Budde WM, Panda-Jonas S. Ophthalmoscopic evaluation of the optic head. Surv Ophthalmol 1999:43:293-320.

6 Jonas JB, Gusek GC, Naumann GOH. Optic disk, cup and neuroretinal rim size, configuration, and correlations in normal eyes. Invest Ophthalmol Vis Sci 1988;29:1151-8.

7 Varna R, Skaf M, Barron E. Retinal nerve fiber layer thickness in normal human eyes. Ophthalmology 1996;103:2114-19. Erratum in: Ophthalmology 1997; 104:174.

8 Alamouti B, Funk J. Retinal thickness decreases with age: an OCT study. Br J Ophthalmol 2003;87:899-901.

9 Zhang J, Quan $\mathrm{H}, \mathrm{Ng}$ J, et al. Some statistical methods for multiple endpoints in clinical trials. Control Clin Trends 1997; 18:204-21.

10 Feise RJ. Do multiple outcome measures require $p$-value adjustment? BMC Med Res Methodol 2002;2:8-12.

11 Quigley HA, Coleman AL, Dorman-Pease ME. Larger optic nerve heads have more fibers in normal monkey eyes. Arch Ophthalmol 1991;109:1441-3.

12 Jonas JB, Schmidt AM, Muller-Bergh JA, et al. Human optic nerve fiber count and optic disc size. Invest Ophthalmol Vis Sci 1992;33:2012-18. 